Meta

Journal des traducteurs

Translators' Journal

\title{
Les publications japonaises sur la traduction : un aperçu
}

\section{Daniel Gile}

Volume 33, numéro 1, mars 1988

Traduction et interprétation au Japon

Translation and Interpretation in Japan

URI : https://id.erudit.org/iderudit/002917ar

DOI : https://doi.org/10.7202/002917ar

Aller au sommaire du numéro

\section{Éditeur(s)}

Les Presses de l'Université de Montréal

\section{ISSN}

0026-0452 (imprimé)

1492-1421 (numérique)

Découvrir la revue

\section{Citer cet article}

Gile, D. (1988). Les publications japonaises sur la traduction : un aperçu. Meta, 33(1), 115-126. https://doi.org/10.7202/002917ar

\section{Résumé de l'article}

Japanese publications on translation are markedly more numerous than Western publications. They are aimed at the general public rather than at professionals or academics, and few are truly scientific or academic. They deal with the Japanese context, with hardly any reference to foreign publications, authors, ideas or translation activities. They are also short-lived and disappear from bookstores and publishers' stocks within a few years.

Theoretical translation texts are "philosophical" rather than scientific. Didactic texts are often aimed at language learners rather than at would-be translators. Linguistic translation texts are more interesting for the insight they give into the Japanese language and its use than for their contribution to translation theory. Texts that criticize published translations are numerous and very popular, something which is rather unique in the world. Many translation books are highly personal and contain numerous anecdotes from their authors' lives. Interpretation books are interesting, as they are more pragmatic than Western texts on the same subject, and address questions that Western publications seldom or never refer to. Machine translation articles are becoming increasingly popular. They tend to be confined to superficial explanations of the operation of systems and to descriptions of commercial products. Truly scientific papers on MT also exist, but their circulation is limited to academic and technical circles.

There are a few periodicals dealing with translation. Most of the articles they carry are written by the same authors and have the same characteristics as the texts described above. On the whole, they are more interesting than translation books, as they are shorter and therefore denser. Articles on translation can also be found in countless books and periodicals on the Japanese language, on linguistics, sociology, public speaking, etc., as well as in weekly and monthly magazines and in other publications.

This paper is followed by a list of Japanese texts on translation and by a list of Western language texts on translation of Japanese or on subjects relevant to the understanding of Japanese translation problems.
Ce document est protégé par la loi sur le droit d'auteur. L'utilisation des services d’Érudit (y compris la reproduction) est assujettie à sa politique d'utilisation que vous pouvez consulter en ligne.

https://apropos.erudit.org/fr/usagers/politique-dutilisation/ 


\section{LES PUBLICATIONS JAPONAISES SUR LA TRADUCTION : UN APERÇU*}

DANIEL GILE INALCO, Paris, France

\section{SUMMARY}

Japanese publications on translation are markedly more numerous than Western publications. They are aimed at the general public rather than at professionals or academics, and few are truly scientific or academic. They deal with the Japanese context, with hardly any reference to foreign publications, authors, ideas or translation activities. They are also short-lived and disappear from bookstores and publishers' stocks within a few years.

Theoretical translation texts are "philosophical" rather than scientific. Didactic texts are often aimed at language learners rather than at would-be translators. Linguistic translation texts are more interesting for the insight they give into the Japanese language and its use than for their contribution to translation theory. Texts that criticize published translations are numerous and very popular, something which is rather unique in the world. Many translation books are highly personal and contain numerous anecdotes from their authors' lives. Interpretation books are interesting, as they are more pragmatic than Western texts on the same subject, and address questions that Western publications seldom or never refer to. Machine translation articles are becoming increasingly popular. They tend to be confined to superficial explanations of the operation of systems and to descriptions of commercial products. Truly scientific papers on MT also exist, but their circulation is limited to academic and technical circles.

There are a few periodicals dealing with translation. Most of the articles they carry are written by the same authors and have the same characteristics as the texts described above. On the whole, they are more interesting than translation books, as they are shorter and therefore denser. Articles on translation can also be found in countless books and periodicals on the Japanese language, on linguistics, sociology, public speaking, etc., as well as in weekly and monthly magazines and in other publications.

This paper is followed by a list of Japanese texts on translation and by a list of Western language texts on translation of Japanese or on subjects relevant to the understanding of Japanese translation problems.

\section{INTRODUCTION}

Un numéro spécial sur la traduction dans un pays donné ne saurait être complet sans une présentation bibliographique. Les publications japonaises sur la traduction semblent être totalement inconnues en Occident; nous avons donc décidé de ne pas dresser une liste bibliographique classique ou une bibliographie sélective et critique, mais de rédiger une synthèse personnelle, présentée du point de vue du traductologue occidental. Par ailleurs, étant donné que la grande majorité des lecteurs de ces lignes ne lisent pas le japonais, nous nous sommes permis de nous affranchir des problèmes techniques liés à la reproduction des caractères japonais en transcrivant les références des différents textes en "romaji» (caractères latins). 
Au-delà de cette synthèse, nous comptons publier des comptes rendus critiques des ouvrages les plus intéressants dans Meta et dans d'autres revues de traductologie. Un premier compte rendu est d'ailleurs paru dans Meta 30-2 (1985), et deux autres sont terminés et doivent être publiés dans un proche avenir.

Pour donner aux lecteurs une idée quantitative de l'importance relative de chaque type de publication et pour leur permettre de rechercher davantage d'informations sur tel ou tel ouvrage par le biais de collègues japonais, nous avons toutefois précisé certaines caractéristiques des textes énumérés sur les listes à la fin de l'article par un système de sigles expliqué plus loin.

\section{PHYSIONOMIE GÉNÉRALE DES TEXTES JAPONAIS}

Si la littérature japonaise commence à être connue en Occident grâce aux traductions de plus en plus nombreuses de romans et autres œuvres, la " barrière linguistique " continue d'isoler les textes documentaires nippons. Chose curieuse, les publications japonaises sur la traduction sont tout aussi isolées que les autres types de textes, car, contrairement aux publications traductologiques occidentales, elles sont rédigées en japonais et non pas dans des langues à vocation plus internationale telles que l'anglais, le français, l'espagnol ou le russe. En réalité, plusieurs caractéristiques distinguent les publications nipponnes des publications occidentales consacrées au même sujet.

\section{Les publications japonaises sont nombreuses}

Par opposition aux publications occidentales sur la traduction, qui se comptent sur les doigts d'une ou deux mains dans la plupart des pays et que l'on trouve difficilement hors des bibliothèques universitaires et assimilées et des grandes librairies, les livres japonais sur la traduction se comptent par dizaines, et sont en partie visibles sur les rayons des librairies de quartier. Les revues Honyaku no sekai (le Monde de la traduction) et Koogyooeigo (l'Anglais technique ${ }^{1}$ ), qui comporte elle aussi une part importante d'articles consacrés à la traduction, se vendent par dizaines de milliers d'exemplaires et sont exposées sur les présentoirs de la plupart des points de vente de revues.

Outre les publications éditées dans des séries consacrées spécifiquement à la traduction, de nombreux articles, monographies et transcriptions de conférences paraissent dans des publications diverses de la presse ou autres (voir à la fin de la section "Genres et thèmes " plus loin). Une telle dispersion rend difficile l'évaluation de l'importance quantitative de ces textes sur la traduction et l'interprétation, mais il ne fait aucun doute qu'ils se comptent au moins par centaines, sinon par milliers.

\section{Les publications japonaises sont destinées au grand public}

Par opposition aux publications occidentales, qui pour la plupart relèvent de la littérature académique ou technique et qui en adoptent le style et la présentation, la grande majorité des livres et revues japonaises sur la traduction s'adressent au grand public, les exceptions étant certains articles paraissant dans des publications périodiques des départements et facultés des universités et certains textes sur la traduction automatique, seul domaine de la traduction où existe une véritable recherche scientifique au Japon.

Sont visés plus particulièrement trois types de lecteurs :

a) Les amateurs de littérature, qui lisent nécessairement des traductions d'ouvrages étrangers, qui sont conscients de l'influence des traductions sur la langue et la littérature japonaises, et qui s'y intéressent dans cette optique. Ce groupe de lecteurs représente une partie non négligeable du public de la revue Honyaku no sekai (voir plus loin). 
Il convient de préciser que le Japon est probablement l'un des pays au monde où se pratique le plus la traduction d'œuvres étrangères, et l'on y trouve des versions nippones de textes de la plupart des grands auteurs de toutes les cultures, que le public japonais, qui lit beaucoup, connaît d'ailleurs assez bien. Il est intéressant de noter que par tradition, le japonais employé dans ces traductions n'est pas le japonais usuel, mais une "langue de traduction "; d'après les témoignages des Japonais que nous avons interrogés à ce sujet, c'est une règle à laquelle échappent très peu de traductions, bien que les théoriciens ne soient pas rares à prôner dans leurs écrits la traduction " naturelle " aboutissant à un langage conforme au génie du japonais. Les amateurs de littérature, ainsi d'ailleurs que les historiens de la langue et les linguistes japonais, s'intéressent donc de très près à la traduction, qu'ils considèrent comme occupant une place importante dans la vie de la littérature et de la langue japonaises.

b) Les appreneurs de langues étrangères, qui ne distinguent pas nécessairement la traduction du thème et de la version scolaires, et qui lisent les ouvrages sur la traduction dans l'optique de l'acquisition des langues. Les auteurs des ouvrages japonais sur la traduction franchissent d'ailleurs eux aussi assez facilement la ligne qui séparerait en Occident les préoccupations propres à la traduction " vraie " de celles de l'apprentissage des langues étrangères.

c) Les aspirants traducteurs, nombreux parmi les jeunes.

En tout état de cause, la quasi-totalité des textes ne semblent pas s'adresser au groupe spécialisé que sont les traducteurs. Leur nature de textes de vulgarisation, d'exercices pratiques de thème ou de version et d'essais ne les prédestine pas à la lecture universitaire, bien qu'une grande partie des auteurs soient eux-mêmes des universitaires.

La plupart de ces textes se vendent très bien, avec des tirages de plusieurs dizaines de milliers d'exemplaires. Ils sont d'ailleurs édités par des maisons d'édition commerciales plutôt qu'universitaires ou techniques. Dès lors, on ne s'étonnera pas d'entendre ceux qui se définissent comme chercheurs, et qui à première vue devraient être comblés parce que disposant d'importants débouchés pour leurs publications, se plaindre d'avoir à sacrifier dans leurs textes la recherche au commercial.

Pour la recherche, il reste les publications des différents départements et facultés universitaires. Quant aux publications commerciales, elles procurent un complément de salaire non négligeable, et ce non seulement par les droits d'auteurs et cachets des articles, mais par la notoriété qu'elles permettent d'acquérir, notoriété qui conduit à des invitations à faire des conférences et à participer à d'autres projets.

\section{Les publications japonaises ne sont pas ouvertes sur l'extérieur}

En effet, contrairement aux textes occidentaux sur la traduction, qui puisent leurs idées et leurs références dans des ouvrages et auprès de personnalités de différents pays et qui sont en grande partie destinés à la communauté internationale des traductologues, les publications japonaises s'adressent exclusivement à un public japonais et ne se reportent quasiment jamais à des théories traductologiques étrangères; tout au plus trouve-t-on ici ou là des références à Nida, Savory ou Mounin dans le domaine de la traduction écrite, et de rares références au Manuel de l'interprète de Jean Herbert dans le domaine de l'interprétation, mais aucun des traductologues occidentaux contemporains et aucune des grandes revues professionnelles ou scientifiques de la traduction (Meta, Babel, Multilingua, Traduire, Lebende Sprachen, etc.) ne sont apparemment connus des auteurs japonais. L'ensemble des publications japonaises sur la traduction ont donc un caractère d'endogamie plutôt étonnant dans un pays qui, dans les domaines du com- 
merce, de l'industrie, de la technologie et même de la littérature, est tant à l'écoute du monde extérieur.

\section{Les textes japonais sur la traduction sont "évanescents"}

Étant donné la destination essentiellement commerciale de ces publications, les tirages semblent prévus pour assurer un chiffre d'affaires maximum. Une fois la période de grand succès passé, les livres disparaissent des rayons, et à quelques exceptions près, même les meilleurs ouvrages deviennent introuvables au-delà d'une dizaine d'années, ce qui rend très difficile la recherche bibliographique.

\section{Les textes japonais sur la traduction sont souvent incorporés dans des ouvrages traitant d'autres domaines}

La traduction n'a pas encore acquis au Japon ses lettres de noblesse en tant que discipline à part entière. Elle constitue plutôt un élément diffusé tous azimuths que l'on retrouve dans d'autres domaines d'étude et de réflexion :

- la critique littéraire, pour des raisons évidentes;

- la langue japonaise, car la traduction a joué un rôle important dans l'évolution du japonais ;

- l'ethnologie et la linguistique comparée, car elle met en valeur les similitudes et différences entre langues et cultures, et les Japonais aiment à réfléchir sur leurs caractéristiques nationales et à les comparer à celles du reste du monde ;

- l'apprentissage des langues étrangères ; en effet, comme il est dit plus haut, les auteurs japonais écrivant sur la traduction s'adressent notamment aux appreneurs de langues étrangères, pour qui la traduction est un débouché possible de l'apprentissage, "débouché " qui commence d'ailleurs souvent bien avant qu'ils aient atteint une maîtrise suffisante de ladite langue, comme en témoignent les nombreux textes critiques mentionnés plus loin. D'où la présencé de livres sur la traduction dans les rayons "langues étrangères" dans de nombreuses librairies; d'où aussi la publication d'ouvrages hybrides, manuels d'anglais médical et de traduction, d'anglais technique et de traduction, d'enrichissement du vocabulaire anglais et de traduction.

Dans la présente synthèse ne sont passées en revue que les publications contenant des segments quantitativement ou qualitativement importants sur la traduction ou relevant suffisamment du domaine de la traduction proprement dite pour être considérées comme allant au-delà des manuels scolaires de thème et de version.

\section{GENRES ET THÈMES}

Étant donné la diversité des textes japonais sur la traduction, leur diffusion à travers une très large gamme de publications et la variété des publics de lecteurs, il est difficile de les soumettre à une classification en partitions nettes. C'est pourquoi dans les pages suivantes sont présentées tour à tour des catégories de textes qui se chevauchent parfois ; il convient de garder cette idée à l'esprit lors de la recherche de catégories ou de thèmes précis dans le présent article.

\section{Essais traductologiques théoriques (ET)}

Cette catégorie de textes, marqués " $\mathbf{E T}$ » dans les listes bibliographiques présentées à la fin du présent article, est quantitativement la plus importante dans les publications japonaises sur la traduction, avec plusieurs dizaines de livres et des centaines d'articles dans la revue Honyaku no sekai et dans des revues de linguistique et de littérature. Dans l'ensemble, elle est assez "philosophique » en ce sens qu'elle présente en termes très généraux les convictions des auteurs sur les rapports entre langues, entre cultures, 
entre langue et culture, sur les difficultés de la traduction liées à ces rapports, et sur les pour et les contre de la « traduction littérale » et de la traduction "libre ». C'est là d'ailleurs une première particularité curieuse et quelque peu archaïque des « traductologues » japónais, qui en partie militent encore pour le littéralisme au nom de la fidélité. Il faut dire que d'autres Japonais sont plus " modernes " que leurs homologues occidentaux, en ce sens qu'ils font appel aux théories modernes de l'information pour analyser la traduction (voir notamment Fujii 1975), ce qui est plus rare chez les Occidentaux - à l'exception des interprètes.

Les textes théoriques sont souvent scolaires, avec quantité d'exemples, en même temps qu'ils sont très personnels, les auteurs expliquant la manière dont ils ont traduit tel ou tel passage dans tel livre comme illustration de leur philosophie.

Dans l'ensemble, par le caractère plutôt superficiel de la présentation des idées qui y sont formulées et étant donné leur redondance les uns par rapport aux autres, ils ne nous semblent pas présenter un très grand intérêt pour le traductologue occidental.

\section{2. 'Textes didactiques (TD)}

Les textes que nous classons dans cette catégorie semblent destinés principalement aux candidats sérieux et moins sérieux à la traduction, c'est-à-dire à de jeunes appreneurs de langues étrangères, à des personnes déjà engagées dans la vie professionnelle à des titres divers qui sont amenées à faire de la traduction et qui recherchent une base méthodologique et parfois théorique, enfin à de vrais " apprentis traducteurs ", essentiellement ceux qui fréquentent (ou suivent par correspondance) les très nombreux cours de traduction proposés par les écoles privées spécialisées.

Les textes didactiques peuvent dans l'ensemble être classés en deux catégories : l'une est assez proche de celle des essais théoriques (ET) évoqués ci-dessus, avec la présentation des principes généraux de la traduction, des conseils sur l'acquisition des langues de travail, quelques informations sur la situation sociale et professionnelle du traducteur, des anecdotes personnelles nombreuses ; l'autre relève davantage du manuel d'exercices pratiques, avec un ensemble de textes japonais à traduire dans la langue occidentale visée (essentiellement l'anglais et le français) ou inversement, des indications terminologiques et méthodologiques, un peu de théorie. C'est dans cette catégorie que l'on a le plus de mal à distinguer les textes scolaires de thème et de version ou d'apprentissage des langues de spécialité des manuels écrits dans une véritable optique de traduction professionnelle.

Les articles didactiques que l'on trouve dans les revues spécialisées, notamment les. numéros spéciaux sur la traduction et l'interprétation du English Journal, sont plus faciles à classer, car plus courts, ils se prêtent moins au " remplissage » et penchent plus nettement vers la didactique pratique ou l'essai.

\section{Textes linguistique (TL)}

Les textes japonais s'appuyant sur la linguistique pour présenter la traduction ou inversement sont très nombreux. Dans l'ensemble, on en distingue trois types.

- Les textes parlant de langue et de traduction dans une optique universelle (TLU), qui se rapprochent de la catégorie ET mentionnée plus haut.

- Les textes parlant de langue et de traduction dans une optique comparatiste japonais-anglais ou japonais-autre langue (TLC).

- Les textes parlant de traduction dans le contexte du japonais (TLJ). Le fait que dans de nombreux manuels de linguistique japonaise, des chapitres entiers soient consacrés à la traduction est une caractéristique japonaise particulière que l'on ne trouve pas dans les ouvrages de linguistique française, anglaise, al- 
lemande ou espagnole, et qui témoigne de la grande importance de la traduction dans l'évolution dynamique du japonais.

Les TL ont en général un aspect très proche de celui des ET, avec une réflexion vulgarisatrice, parfois dogmatique et rarement originale sous l'angle de la traduction. Sur le plan traductologique, ils n'apportent donc qu'une modeste contribution. Ils présentent toutefois un certain intérêt sur le plan de la linguistique contrastive, car ils mettent en valeur des similitudes et différences entre le japonais et les langues occidentales avec des exemples précis. Dans l'ensemble, ces textes, ainsi d'ailleurs que des textes linguistiques purs, sont donc intéressants pour le traductologue occidental, non pas en tant que textes traductologiques, mais comme matière brute lui permettant de pousser sa réflexion au-delà de l'horizon purement occidental.

\section{Textes critiques (TC)}

Une autre particularité de la traductologie japonaise est la popularité des livres consacrés entièrement à la critique des traductions parues au Japon. D'une manière générale, l'on trouve dans ces textes deux critiques : l'une porte sur le caractère peu naturel du japonais employé dans les traductions par rapport au japonais "spontané " ou " authentique " (voir plus haut), et l'autre sur les erreurs commises dans des traductions publiées. On est d'ailleurs surpris de voir que ces erreurs sont souvent des fautes de compréhension très grossières qui témoignent soit d'un niveau linguistique très nettement insuffisant en langue étrangère chez les traducteurs, soit de conditions de travail des traducteurs, éditeurs et imprimeurs néfastes à la qualité - c'est surtout à cette deuxième source de lacunes que s'attaque l'ancien père jésuite belge W.A. Grootaers dans son très célèbre best-seller Goyaku (les Erreurs de traduction).

\section{Textes anecdotiques (TA)}

Comme il est dit plus haut, les livres sur la traduction sont des produits populaires qui ont un effet multiplicateur sur la renommée de leurs auteurs qu'ils transforment en vedettes nationales. Étant donné ce caractère populaire des textes et le vedettariat qui y est lié, la personnalisation des livres est appréciée des lecteurs, ce qui conduit les auteurs non seulement à les parsemer d'anecdotes personnelles, mais aussi à écrire des bibliographies ou quasi-bibliographies, voire à les faire suivre par des deuxièmes parties ou par de nouvelles versions : le livre de Nishiyama Sen Tsuuyakujutsu to watakushi (la Technique d'interprétation et moi), publié en 1979, n'est en fait qu'une nouvelle version de Tsuuyakujutsu (la Technique d'interprétation), publié en $1970 \mathrm{chez}$ un autre éditeur, les deux livres contenant le même mélange de philosophie générale de l'interprétation et d'anecdotes autobiographiques, les mêmes pour la plupart ; autre exemple, le best-seller de Masumi Muramatsu Watakushi mo eigo ga hanasenakatta (titre anglais : I Couldn't Speak English Either), un recueil d'anecdotes autobiographiques publié en 1978, a été suivi un an après d'un deuxième recueil du même type intitulé en toute franchise Watakushi mo eigo ga hanasenakatta - zoku (I Couldn't Speak English Either : A Sequel).

Autre type de textes anecdotiques, les nombreuses interviews et autres articles où des traducteurs connus et moins connus racontent leurs débuts de carrière, leur vie quotidienne et leurs préoccupations personnelles.

Ces textes peu prétentieux donnent au lecteur un aperçu historique et sociologique des conditions de travail des traducteurs japonais depuis la Deuxième Guerre mondiale et ne sont donc pas inintéressants, bien qu'il ne faille pas espérer en tirer d'importantes quantités d'informations scientifiquement ou théoriquement exploitables. 


\section{Textes « factuels" (TF)}

Si les textes théoriques et didactiques japonais peuvent paraître quelque peu décevants pour le traductologue occidental, il existe un domaine où les textes japonais sont incontestablement supérieurs aux publications occidentales : celui des textes "factuels ». Dans le numéro spécial que consacre tous les ans The English Journal à la traduction (pendant quatre ans, il en avait fait de même pour l'interprétation) figurent de nombreuses pages contenant des données abondantes sur différents aspects pratiques de la traduction ; tarifs et rémunérations, noms, adresses, spécialités des agences de traduction, listes d'écoles, leurs effectifs, programmes, enseignants, frais de scolarité et conditions d'admission. Les revues présentent également des reportages sur les cours de traduction, des renseignements biographiques et personnels sur des traducteurs connus (y compris leurs adresses et numéros de téléphone personnels), des interviews avec des traducteurs, des éditeurs, des directeurs d'agences de traduction, des comparaisons entre systèmes de traitement de texte et informations sur des systèmes de traduction automatique, des listes d'ouvrages traduits récemment, etc. L'on peut donc dire que si au Japon la traduction est quasiment exclue du domaine de la recherche scientifique, elle y bénéficie d'une couverture journalistique remarquable et inimaginable en Occident. On notera l'ironie de la contradiction : alors que le traductologue scientifique occidental a de considérables difficultés d'accès à des faits qui lui permettraient de dégager des hypothèses et de les vérifier, l'accès à ces mêmes faits est aisé au Japon, mais il ne profite à personne faute de traductologues scientifiques désireux de les exploiter.

\section{Textes sur l'interprétation (TI)}

C'est dans ce domaine que les publications japonaises sont les plus intéressantes pour les traductologues occidentaux. On trouve en effet dans la douzaine de livres japonais sur l'interprétation des points de vue différents de ceux des théoriciens occidentaux de l'interprétation : ces derniers semblent s'être attachés, surtout dans les années 60 et 70 , à justifier, voire à renforcer le prestige initial de ces "phénomènes " qu'étaient les premiers interprètes, et démontent les mécanismes de l'interprétation dans un univers absolu et parfait ; les Japonais, eux, et notamment Nishiyama Sen (1969), portent un regard lucide sur l'environnement réel dans lequel ils travaillent. C'est ainsi qu'ils n'hésitent pas à reconnaître les limites de leur profession : ils admettent que toute communication comporte une perte d'information, que l'interprète ne peut tout interpréter, chargent l'orateur d'une partie de la responsabilité de la transmission de son message à travers l'interprète. En outre, par des exemples nombreux, ils fournissent des illustrations des difficultés de la communication à travers l'interprétation dans un cas où les delux langues - et les deux cultures - en présence sont fort éloignées, alors que la plupart des publications occidentales dans ce même domaine portent sur des cas où les interlocuteurs sont culturellement voisins. On trouve donc dans les textes japonais une démarche réaliste et pratique, ainsi que des éléments susceptibles de bousculer un certain nombre d'idées reçues occidentales (il conviendrait peut-être aussi de bousculer quelques-unes des idées reçues japonaises, telles que la présumée possibilité d'interpréter par transcodage entre langues occidentales en raison de leur ressemblance).

\section{Traduction automatique (AUT)}

La TA est le seul domaine où les textes véritablement scientifiques côtoient les ouvrages de vulgarisation qui composent l'essentiel de la masse des textes japonais sur la traduction. Comme il est dit plus haut, c'est apparemment aussi le seul secteur de la traductologie où s'exerce une véritable activité de recherche au Japon, peut-être en raison de ses retombées technologiques et économiques et de la prise en charge des travaux par 
des informaticiens et des électroniciens. C'est également le seul domaine traductologique où existe une véritable coopération entre le Japon et le reste du monde, avec des projets nippo-français et nippo-allemands entre autres. Il est vrai que les textes scientifiques sont surtout réservés aux comptes rendus de colloques et aux organes universitaires, et que les textes sur la TA qui trouvent leur chemin jusque dans les librairies sont plutôt des initiations vulgarisatrices, des reportages et des comparaisons de performances. Les textes sur la TA deviennent de plus en plus nombreux dans les revues de traduction et sont présents dans la plupart des numéros les plus récents ; citons par exemple un numéro spécial sur la TA de Honyaku no sekai (1984:9/9), et, plus surprenant mais caractéristique de l'intérêt général que suscite ce thème au sein du public japonais, le numéro spécial sur la TA de Jijieigo - The Study of Current English (mai 1986 : 41/2).

De manière générale, les textes japonais sur la $T A$ ne présentent pas un caractère original sur le plan théorique ; ils ne sont toutefois pas inintéressants dans la mesure où ils évoquent des problèmes et solutions en matière d'analyse linguistique du japonais à des fins de traitement informatique.

\section{LES REVUES COMMERCIALES}

\section{Honyaku no sekai:}

La seule (et grande) revue consacrée spécifiquement à la traduction est Honyaku no sekai. Édité par la maison Nihonhonyakkayooseisenta, qui est avant tout une école privée de traduction, ce mensuel de 160 pages a une vocation essentiellement commerciale. L'équipe de rédaction se compose de trois journalistes, qui ne sont d'ailleurs pas traducteurs, et les contributions extérieures sont en principe écrites sur demande et à titre onéreux par des personnalités japonaises du monde de la traduction. Chaque numéro de Honyaku no seikai comporte des critiques de traductions, des articles pédagogiques (exercices commentés de traductions, conseils sur différents genres de textes, etc.), des listes d'ouvrages parus récemment, des interviews de personnalités du monde de la traduction japonais, et de très nombreuses pages de publicité pour des livres, des écoles de traduction et des écoles de langues; chaque numéro contient également un dossier spécial (traduction de films, traduction de bandes dessinées, comment polir son japonais, les dictionnaires, etc.).

Dans la mesure où les articles de fond sont rédigés par les mêmes auteurs que les livres mentionnés plus haut, Honyaku no sekai offre un assez bon panorama des textes traductologiques japonais, avec les mêmes mérites et les mêmes défauts que les livres. Les interviews sont souvent intéressantes, et les articles factuels sont des sources d'information utiles. À noter un numéro spécial hors série intitulé "Honyakkasakusesujiten " (titre anglais : "Access to Success") datant de 1985 et comprenant outre des interviews, des critiques de traduction et des conseils, des informations diverses sur les écoles, les tarifs, les systèmes de traitement de textes, les ouvrages japonais traduits en langue étrangère. Ce numéro spécial est très semblable aux numéros spéciaux de The English Journal (voir ci-dessous).

\section{Koogyooeigo ("L'anglais technique ")}

Il s'agit d'une autre grande revue qui est consacrée à l'anglais technique sous trois aspects : l'anglais en tant que langue technique, la traduction japonais-anglais et la traduction anglais-japonais. Son intérêt réside dans ses nombreux textes techniques avec traduction, que l'on ne trouve guère dans Honyaku no sekai, ni d'ailleurs dans la plupart des autres publications sur la traduction. 


\section{The English Journal}

Cet important mensuel publie de nombreux numéros spéciaux hors série sur l'anglais et les études d'anglais, mais aussi, depuis 1980, un numéro spécial hors série annuel sur la traduction, et entre 1981 et 1984 sont parus quatre numéros spéciaux analogues sur l'interprétation. Ces numéros spéciaux sont semblables dans leur présentation au "Honyakkasakusesujiten" de Honyaku no sekai, mais avec plus de 200 pages et un matériel plus soigneusement sélectionné.

Pour le traductologue occidental, ces numéros spéciaux nous semblent être la meilleure source d'information écrite sur la traduction au Japon, notamment en matière d'interprétation, où il n'existe pas d'autres périodiques.

\section{Autres revues commerciales}

En un an de recherches dans les bibliothèques et librairies et de demandes d'information auprès de collègues japonais, nous n'avons pas trouvé d'autres revues commerciales sur la traduction, à l'exception d'un numéro 6 unique de la revue trimestrielle $\mathrm{Ho}$ nyaku de la maison Mikibo datant de mars 1985, dans une librairie spécialisée dans les livres d'occasion. Cette revue, inconnue de l'ensemble de nos informateurs japonais, a dû avoir une durée de vie très courte.

Par contre, d'autres revues non liées à la traduction présentent des numéros spéciaux occasionnels ou des articles sur la traduction : le numéro spécial de Jijieigo sur la traduction automatique a été mentionné plus haut; la revue de linguistique Gengo a consacré ses numéros $13 / 5$ et $13 / 6$ (1984) à la traduction; le numéro $1 / 4(1972)$ du mensuel de linguistique Gekkangengo est également un numéro " traduction "; la revue littéraire Doojidai a consacré son numéro 38 (septembre 1981) à la traduction poétique ; un article de Masao Kunihiro lié à l'interprétation est paru dans The Japan Interpreter 8/1 (1973), et des recherches suffisamment longues permettraient certainement de découvrir bien d'autres articles sur la traduction dans des revues diverses.

\section{AUTRES PUBLICATIONS}

Les périodiques commerciaux ne sont pas les seuls qui soient consacrés à la traduction. Le Nihonhonyakkayooseisenta, éditeur de Honyaku no sekai, publie également Honyakujin et le distribue aux élèves de l'école. D'autres écoles de traduction ou d'interprétation, dont certaines portent le titre d'" association ", ont leurs propres bulletins de liaison où l'on trouve davantage de renseignements pratiques et d'interviews avec des dirigeants d'écoles et directeurs de sociétés de traduction que d'articles de fond. À noter cependant des articles de fond nombreux et intéressants dans le modeste JAT Bulletin, organe mensuel de la Japan Association of Translators (JAT), une véritable association de traducteurs. C'est d'ailleurs le seul organe de ce type à être rédigé en anglais, avec de rares articles en japonais. Notons à ce propos l'existence aux États-Unis de Atarashii, un bulletin analogue du Japanese Interest Group de la American Translator's Association qui prend la succession du bulletin Technical Japanese Translation de Donald Philippi. Il n'existe apparemment pas de publications équivalentes en Europe ou dans d'autres parties du monde.

Les publications universitaires contiennent elles aussi des articles sur la traduction, surtout dans un contexte littéraire ou dans l'optique de l'apprentissage des langues, mais aussi dans des contextes de communication, de sociologie, d'ethnologie, de politique et d'économie, car il existe des traducteurs et interprètes parmi les universitaires japonais spécialisés dans ces disciplines qui y présentent les problèmes de la communication interculturelle à travers leur expérience de traducteurs et d'interprètes. Notons que la publication dans ces organes compte apparemment davantage dans les carrières universitaires que la publication dans les sevues internationales, ce qui expliquerait en 
partie l'absence des Japonais dans ces revues ; une autre explication, valable même pour les traducteurs, est d'ordre linguistique...

La NHK, organe national de radio et télédiffusion, propose des émissions éducatives et culturelles qui s'accompagnent de publications mensuelles, notamment dans le domaine de l'apprentissage des langues. Dans le cadre des émissions de "conversation anglaise " ont été diffusées puis publiées plusieurs conférences et interviews sur l'interprétation.

Enfin, différentes publications de maisons d'édition et autres sociétés et institutions contiennent des transcriptions de discours, conférences et entretiens sur la traduction et l'interprétation, et il n'est pas rare que des quotidiens, hebdomadaires et mensuels ordinaires contiennent des articles sur ces deux sujets.

L'intérêt de la plupart de ces textes est modeste. En conséquence, et compte tenu de leur grande dispersion qui les rend difficiles à trouver, nous n'en présentons pas une liste séparée ici. Certains apparaissent toutefois sur la liste $\mathrm{n}^{\circ} 2$ (textes rédigés dans des langues occidentales).

\section{LISTES BIBLIOGRAPHIQUES}

\section{Liste 1 : Livres sur la traduction en japonais}

\section{Abréviations :}

AUT : traduction automatique

ET : texte théorique

LIT : optique littéraire ou historico-littéraire

TA : texte anecdotique

TC : texte critique

TD : texte didactique

$\mathrm{TF}$ : texte factuel ou pratique

TH : texte historique

TI : texte sur l'interprétation

TLA : optique apprentissage de l'anglais

TLC : optique linguistique comparative

TLJ : optique linguistique japonaise

TLU : texte linguistique, optique " universelle"

ANZAI, Tetsuo (1982) : Honyakueibunpo, Nihonhonyakkayooseisenta [ET, TLA].

BEKKU, Sadanori (1975) : Honyaku wo manabu, Yashioshuppansha [ET].

BEKKU, Sadanori (1979) : Honyakudokuhon, Kodanshagendaishinsho [ET, TD].

BEKKU, Sadanori (1980) : Honyaku no shohoo, Japan Times [ET, TD].

BEKKU, Sadanori (1981) : Goyaku meiyaku kettenhonyaku, Bungeishunju [TC].

BEK KU, Sadanori (1982) : Kodawarihen honyakunooto, PHP Kenkyuusha [ET, TC].

BEKKU, Sadanori (1983) : Eibun no honyaku (titre anglais : Translating English Literature), Taishuukanshoten [TD, TLC, TLA].

BEKKU, Sadanori (1986) : Konna honyaku ni dare ga shita, Bungeishunshu [TC].

BEKKU, Sadanori (?) : Zoku Goyaku meiyaku kettenhonyaku, Bungeishunshu [TC]

BEKKU, Sadanori ( ?) : Koona honyaku yomitakunai, Bungeishunka [TC].

FUJI, Akio (1975) : Nihonjin no honyaku prosesu - nihongo kara eigo he, Wasedadaigakushuppanbu [ET].

FUKUII, Haruhiro et Tasuke ASANO (1961) : Eigotsuuyaku no jissai, Kenkyuusha [TI]

FUKUDA, Tsuneari (1966) : "Honyakuron", dans Gendaieigokyooikukooza, vol. 19, Kenkyuusha [ET, TLA].

FUTABATEISHIMEI (1906) : "Yo ga honyaku no hyoojun", in Seikoo, republished in Futabateishimei zenshuu, dai 4 kan, Iwanami shoten 1965 [LIT].

GUROTASU, Shibata (trad.) (1967) : Goyaku, Sanseidoshinsho [TC].

HIGASHIDA, Chiaki (1981) : Chokuyaku to iu mei no goyaku, Nanundo [ET, TC].

IKEDA, Magako (1982) : Honyaku, Iwanamishoten [ET].

INTERPRESS (1982) : Fukugyoo to shite no honyaku [TF]. 
ISOYA, Tabashi (1980) : Honyaku to bunka no kigooron, Keisoshoboo [ET]

ITO, Hitoshi (1933) : "Honyaku no kenkyuu ", in Eigœibungakukooza, Shineibeibungakusha [LIT].

IWABUCHI, Etsutaro (1960) : Akubun, Nihonhyooronsha [TC].

IWANAMISHOTEN (1982): Honyaku (recueil d'essais par 44 auteurs). Iwanamishoten [ET].

KAWAI, Sin (1978) : Honyaku ga umaku naru hayakujitsu koosu, Asahi Evening News [TD]

KAWAMORI, Yoshizo (1961) : "Honyakubungaku", dans Kindaibungakukanshookooza, vol. 21, Kadokawashoten [ET, LIT].

KODAKA, Nobumitsu (1985) : Honyaku to iu shigoto, Presidentosha [TA]

KONO, Ichiro (1975) : Honyakujootatsuhoo, Kodanshagendaishinsha [TD].

KONO, Ichiro (1982) : Honyakukyooshitsu, Kodanshagenjoshinsho [TD].

KUNIHIRO, Masao, Sen NISHIYAMA, Nobuo KANAYAMA (1969) : Tsuuyaku, Eikaiwa kara doojitsutsuuyaku made, Nihonhoosooshuppankyookai [TI].

KURITA, Akiho (1975) : Tsuuyaku ni naru ni wa, Naruniwa books [TI, TF].

MAKINO, Tsutomu (1981) : Honyaku no gihoo, Wasedadaigakushuppanbu [ET].

MATSUMOTO, K. and K. MUKAI (1976) : Eigotsuuyaku he no michi, Nihontsuuyakukyookai [TI, ET].

MATSUMOTO, Yasuhiro et Irene MATSUMOTO (1982) : Ima sugu yaku ni tatsu honyaku no 84 kotsu, Nihonhonyakkayooseienta [TD].

MATSUMURA, Tatsuo (1978) : Honyaku no ronri, Tamagawadaigakushuppanbu [ET].

MILWARD, Peter (1965) : "Honyakuron", dans Gendaieigokyooikukooza, vol. 9, Kenkyuusha [ET, TLA].

MIYOSHI, Hiroshi (1980) : Sugu tsukameru eigohonyaku no kotsu, Asahishuppansha [TD].

MORI, Tooru (1982) : "Honyaku - akubun no ryoorihoo", Japan Times [TC].

MORI OGAI (1914) : "Honyaku ni tsuite", first published in Gendainijuumeikabunshoosakuhookoowa, Tokyo mangeido, republished in Moriogaizenshu, dai 26 kan, Iwanamishoten (1973).

MORITA, Sohei (1933) : Honyaku no riron to jissai, Shineibeibungakusha [ET].

MURAMATSU, Masumi (1978) : Watakushi mo eigo ga hanasenakatta (titre anglais : I couldn't speak English either), Saimarushuppansha [TA, TI].

MURAMATSU, Masumi (1979) : Watakushi mo eigo ga hanasenakatta - zoku (titre anglais : I couldn't speak English either - a sequel, Saimarushuppansha [TA, TI].

NAGAO, Makoto (1986) : Kikaihonyaku wa doko made kanoo ka, Iwanamishoten [AUT].

NAKAMURA, Yasuo (1973) : "Honyaku no gijutsu", Chukoshinsho [ET]

NAKAMURA, Yasuo (1983) : "Honyaku wa doko made kanoo ka", Japan Times [ET].

NAKAMURA, Yasuo (1982): Honyaku no hiketsu, Shinchosensho [TLA].

NAKANO, Yoshio (1943) : "Honyakubungakuron ", taishoshowajidai, dans Kindainihonbungakenkyuu showabungakusakkaron, vol. 2, Shogakkan [LIT].

NII, Itaru (1941) : "Honyakuron", dans Kokugobunkakooza, Asahishinbunsha [LIT]

NISHIYAMA, Sen (1970): Tsuuyakujutsu, Jitsunichishinsho [TI, TA].

NISHIYAMA, Sen (1979) : Tsuuyakujutsu to watakushi, Purezidentosha [TI, TA].

NOGAMI, Toyoichiro (1938) : Honyakuron, Iwanamishoten [ET].

OGATA, Tamotsu (1975) : "Honyaku no kotsu", Japan Times [TLA].

OTA, Saburo (1959) : "Honyakubungaku", dans Iwanamikooza - nihonbungakushi, vol. 14, Iwanamishoten [LIT].

SAWAMURA, Tonazino (1935) : Yakudoku to honyaku, Kenkyuusha [LIT].

SEIDENSTICKER, E.G. et T. ANZAI (1983) : Nihonbun no honyaku (titre anglais : Translating Japanese Literature), Taishuukanshoten [TLC].

SHIBATA, Masaharu (1982) : Dare ni demo dekiru tsuuyakunouhau, Gendaishuppan [TI, TF].

SHIMADA, Kinji (1951) : "Honyakubungaku ", dans Nihonbungakukyooyookooza, vol. 13, Shibundo [LIT].

SHINODA, Yoshiaki (1972) : Koogyooeigo (titre anglais : How to Translate Technical English), Asahishuppansha [TLA, TD].

SHUMATA, Natsuo (1979) : Honyaku no jooshiki, Yashioshuppansha [ET]

SUGIMOTO, Tsutomu (?), Nihonhonyakugoshi no kenkyuu, Yazakashoboo [TH].

TAKAHASHI, Masao (1964): Goyaku - Daigakukyooju no atama no hodo, Yukishobo [TC].

TAKAHASHI, Yasukuni (1982) : "Nihongo wo migaku honyakujutsu", Babel [TD].

TAKEUCHI, Kenji (1982): Goyaku, Chobunsha [TC].

TAKEYOSHI, Jiroo (1984) : Chugokugohonyaku-tsuuyaku handbook, Tohoshoten [TF].

YANABU, Akira (1972) : Honyakugo no ronri, Hoseidaigakushuppankyoku [ET].

YANABU, Akira (1976) : Honyaku to wa nanika-nihongo to honyakubunka, Hoseidagakushuppankyoku [ET].

YANABU, Akira (1977): Honyaku no shisoo, Heibonsha [ET].

YANABU, Akira (1978a) : "Honyaku no mondai ", Iwanamikooza, dans Nihongo bekkan nihongokenkyuu no shuuhen, Iwanamishinten [ET, TLJ].

YANABU, Akira (1978b) : Honyakubunka wo kangaeru, Hoseidaigakushuppankyoku [ET, LIT].

YANABU, Akira (1983) : Honyakugakumonhihan, Nihonhonyakuyooseisenta [TC]. 
YANAGIDA, Izumi (1928) : Meïi no honyakubungakukenkyuu, Schinchosha [LIT, TH].

YANAGIDA, Izumi (1943) : "Honyakubungakuron - meijijidai ", dans Kindainihonbungakukenkyuu, shoowabungakusakkaron, vol. 2, Shogakkan [LIT]

YANAGIDA, Izumi (1961) : Meijishokihonyakubungaku no kenkyuu, Shunjusha [TH]

YANASE, Naoki (1980) : Honyakukomaribanashi, Hakuyosha [TA]

YOKOI, Tadao (1971) : Goyaku akuyaku no byoori, Gendaijaanarizumushuppankai [TC]

YOSHITAKE, Yoshinori (1959): Meijitaishoono honyakushi, Kenkyusha [TH].

Liste 2 : Textes sur la traduction du japonais en langues occidentales et textes de linguistique japonaise en anglais susceptibles d'intéresser les traductologues

occidentaux.

ATARASHII : Bulletin du Japan Interest Group de la American Translators Association.

BERNIER, Normand (1966) : "La traduction du japonais ", META, 11-3, pp. 79-84.

CONDON, John and Mitsuko SAITO (1974) : Intercultural Encounters with Japan, The Simul Press.

GILE, Daniel (1983) : "Initiation à la traduction scientifique et technique japonais-français à l'INALCO la recherche d'une optimisation des méthodes ", Bulletin des anciens élèves de l'INALCO, juin.

GILE, Daniel (1984) : " La logique du japonais et la traduction : un exemple ", Contrastes, $n^{\circ}$ 9, novembre

GILE, Daniel (1985a) : "La logique du japonais et la traduction des textes non littéraires", Babel, 31-2, pp. 86-94.

GILE, Daniel (1985b) : "Goyakuakuyaku no byoori " (compte rendu), Meta, 30-2, juin.

GILE, Daniel (1986) : "La reconnaissance des kango dans la perception du discours japonais ", Lingua, $70 / 2-3$.

GORHAM, Don C. (1984) : "Workshop in Japanese-English Translation ", in Silver Tongues, Proceedings of the 25th Annual Conference of the American Translators Association, New York, September 19-23, Edited by E. Newman Medford, N.J., Learned Information Inc., $478 \mathrm{p}$.

JAT Bulletin, Bulletin de la "Japan Association of Translators".

KODAMA, James Hisao (?) : "Shakespearian Translation and the Japanese Language - Some Difficulties", Shakespeare Translation, no 9, pp. 19-32.

KORETSUNE, Takashi (1977) : "The Quality Control Concept in Technical Translation in Japan ", in Paul A. Horguelin (Ed.), Translating, a Profession, Montréal, Actes du viII congrès mondial de la Fédération internationale des traducteurs.

KUNIHIRO, Masao (1973) : "Indigenous Barriers to Communication ", The Japan Interpreter, 8-1, pp. $1-13$

MILWARD, Peter (?) : "On Translating Shakespeare into Japanese », Shakespeare Translation, nº 8, pp. $17-24$

MIZUTANI, Osamu (1981) : "The Spoken Language in Japanese Life", The Japan Times.

NISHIYAMA, Sen (1983) : "Speaking Someone Else's Mind", Winds, The Inflight Magazine of Japan Airlines, 5-7, December.

PHILIPPI, Donald : Technical Japanese Translation, bulletin

SUZUKI, Takao (1973) : "Japanese and the Japanese ", Words in Culture, Iwanami shoten.

TANAKA, Chiharu (1979) : "Aperçu sur l'histoire de la traduction au Japon ", Babel, 25-3, pp. 127-130.

YEOMANS, R. (1973) : "Translation in Japan : Notes on a Short Visit ", Babel, pp. 158-160.

Remerciements * Messieurs Osamu KANAMORI et Michihiro NOHARA ont apporté à l'auteur une aide précieuse en lisant et en commentant une partie des textes japonais qui sont à la base du présent article. Qu'ils soient remerciés ici de leur collaboration. 